\begin{tabular}{|c|c|}
\hline Title & Dynamical properties of atom-like emissions from single localized states in ZnCdS ternary mesa shaped structures \\
\hline Author(s) & Kumano, H.; Hitaka, Y.; Suemune, I. \\
\hline Citation & $\begin{array}{l}\text { physica status solidi (b), 241(3), } 503-506 \\
\text { https://doi.org/10.1002/pssb.200304270 }\end{array}$ \\
\hline Issue Date & 200403 \\
\hline Doc URL & http:/hdl.handle.net/2115/59638 \\
\hline Rights & $\begin{array}{l}\text { This is the pre peer reviewed version of the following article: phy sica status solidi (b)V olume } 241 \text {, Issue 3, pages } 503 \\
506 \text {, March 2004, which has been published in final form at } \\
\text { http://onlinelibrary.wiley .com/doi } 10.1002 / \text { pssb.200304270/abstract. }\end{array}$ \\
\hline Type & article (author version) \\
\hline File Information & pss2004.pdf \\
\hline
\end{tabular}

Instructions for use 


\title{
Dynamical properties of atom-like emissions from single local- ized states in ZnCdS ternary mesa-shaped structures
}

\author{
H. Kumano*, Y. Hitaka, and I. Suemune
}

Research Institute for Electronic Science (RIES), Hokkaido University, Kita-12, Nishi-6, Kita-ku, Sapporo 060-0812, Japan

Received zzz, revised zzz, accepted $z z z$

Published online $z z z$

PACS 78.55.Et, 78.67.-n, 71.35.-y, 42.50.Ct

Dynamical properties of exciton localization into spatially isolated localized states with atom-like density of states caused by alloy potential fluctuations were examined. Submicron-sized mesa-shaped structures were prepared to resolve the contribution of single localized states to the optical spectra, which makes it possible to observe atom-like emission lines and to discuss the dynamical properties of exciton energy relaxations by employing micro-timeresolved PL measurements. It will be shown that in the samples with high exciton energy transfer efficiency, photogenerated excitons can relax into the spatially isolated localized centers with strong zero-dimensional nature, which leads to the limited number of atom-like emission lines.

(1)

\section{Introduction}

In the past few years, studies on quantum information science have attracted a huge interest for the realization of single quantum devices such as single photon emitters (SPE)[1-3]. Quantum information theory predicts that the SPE devices will have a possibility of overwhelming functions that are far beyond the capability of classical ones, that is, completely secure quantum cryptographic key distribution [4]. The quantum key distribution (QKD) can provide a hardware-based solution to network security issues based on indivisibility of single photons. In the $\mathrm{QKD}$, generation of single photons relies on strongly attenuated coherent light as a single photon source. However, this source can also create multiphotons due to Poisson distribution in the coherent light, which makes it vulnerable to eavesdropping by photon splitting [5,6]. Exciton emission in zero-dimensional (0-D.) system is promising as an alternative light source due to their capability of delivering nonclassical deterministic single photons on demand. So far, researches on single photon emission based on 0-D. exciton states are almost exclusively focused on InAs related quantum dots (QDs). However, the operation temperature will be limited in low temperature range and furthermore, the conventional III-V QDs cannot reach into a strong coupling regime[7] which is inevitable for the coherent control.

In comparison to the III-V, wide bandgap semiconductors have higher confinement potential and larger oscillator strength. Therefore they are promising for the higher temperature operation and enhanced exciton-photon interaction to reach into the strong coupling regime. Very recently, we have reported atom-like discrete sharp emission lines from 0-D. localized exciton states in ZnCdS alloy semiconductors [8]. In this case, emitting photon energy can be tailored by tuning the alloy composition and favorable for the future effective coupling with discrete photon modes in microcavities. So far, stationary optical properties of the observed atom-like emission lines from 0-D. localized states in the alloy semiconductors were discussed in ref. 8, however, the dynamical properties of exciton relaxation into the localized states are less well understood. Exciton dynamics including the exciton energy relaxation and

\footnotetext{
* Corresponding author: e-mail: kumano@es.hokudai.ac.jp, Phone/Fax: +81-11-706-2898
} 
capture rate into the well-isolated localization states which offers the highly 0-D. states are essential issues to be clarified since these properties will possibly determine the whole response time of the practical SPE.

In this paper, dynamical properties of exciton relaxation into spatially isolated 0-D. localized centers are discussed. Selectively grown mesa-shaped structures and spatially-resolved micro-time-resolved PL ( $\mu$-TRPL) measurements were employed for this purpose. It will be shown that photogenerated excitons relax into spatially isolated localized centers within 40 ps after excitation, which shows effective exciton population into the single localized states. It is also revealed that limited number of atom-like emission lines are possible in the samples with high exciton transfer efficiency.

\section{Experimental procedure}

The samples studied were prepared by metalorganic molecular-beam epitaxy on $n^{+}$-GaAs (001) substrates at $380^{\circ} \mathrm{C}$. ZnCdS/MgS short period superlattices (SPSL) with respective ZnCdS and MgS layer thicknesses of $1.0 \mathrm{~nm}$ and $0.8 \mathrm{~nm}$ were selectively grown in the mask openings patterned by electron beam lithography. Mixed-crystal formation is expected to be well suppressed since the $\mathrm{Mg}$ incorporation into the ZnCdS layers will distinguishably increase the emitting energy due to very high band-gap of MgS, which is not the case for our optical measurements. Further details on the mask patterning procedures and the selective growth are given in ref. 9. Typical AFM image of a selectively grown area reveals a formation of mesa-shaped structure as is illustrated in the inset of Fig. 1. In order to pick up PL signal exclusively from a single mesa structure, $\mu$-TRPL measurements with a x100 microscope objective lens were carried out at the temperature of $20 \mathrm{~K}$ using a frequency-doubled Ti:Sapphire laser as an excitation source. The pulse duration and the repetition frequency were $100 \mathrm{fs}$ and $82 \mathrm{MHz}$, respectively, and the excitation density per pulse was approximately $1 \mu \mathrm{J} / \mathrm{cm}^{2}$ at the wavelength of $400 \mathrm{~nm}$. The synchro-scan streak camera was used as a detector and the spectral and temporal resolutions of the total system were $0.6 \mathrm{~nm}$ and $20 \mathrm{ps}$, respectively.
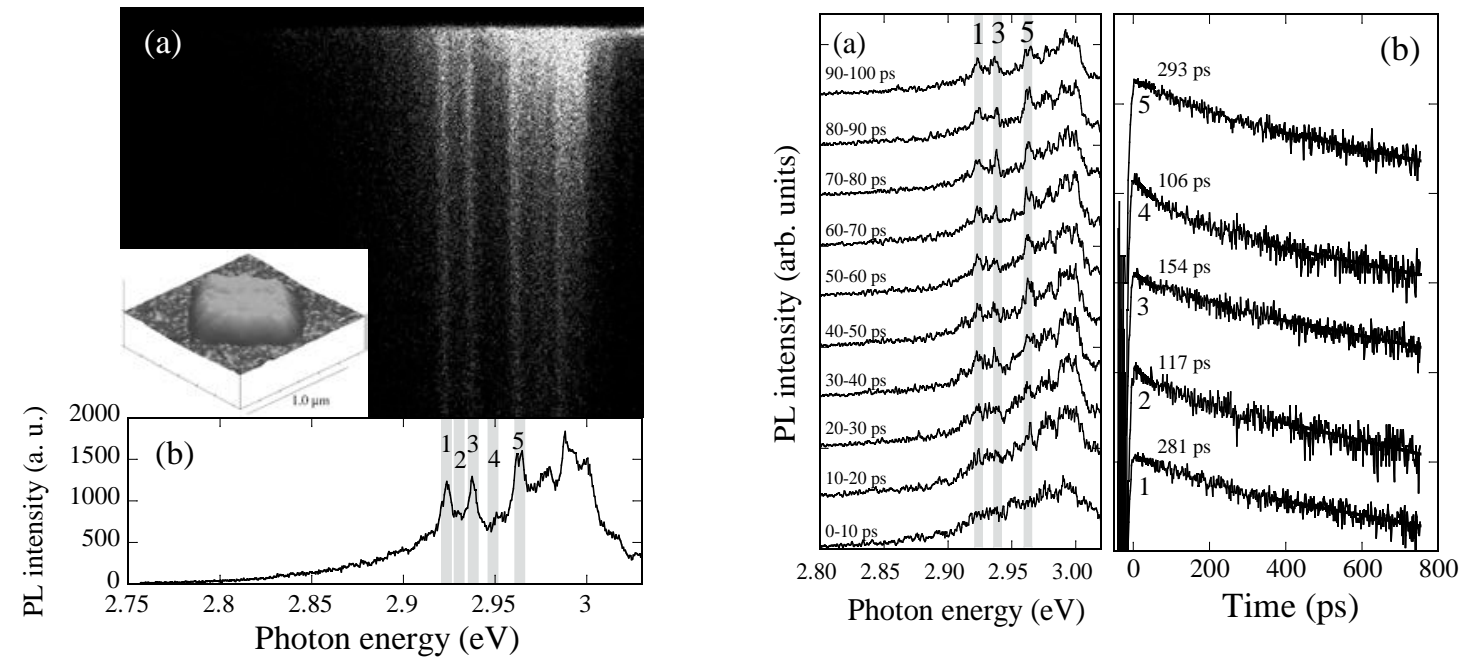

Fig. 1 (left) (a) Streak image of single selective-grown ZnCdS/MgS SPSL structure. Full vertical time axis corresponds to 800 ps. (b) Time-integrated PL spectrum. Several sharp emission lines are resolved in shaded area of 1,3, and 5. AFM image of a typical selectively grown mesa structure is also shown in the inset. 
Fig. 2 (right) (a) Transient PL spectra extracted from Fig. 1(a) with 10 ps time width. (b) PL decay curves detected at each shaded energy range given in Fig. 1(b). Decay times reduced by time domains of $t<50 \mathrm{ps}$ after excitation pulses are also shown.

\section{Results and Discussions}

In Figs. 1, a streak image obtained from the selectively grown ZnCdS/MgS SPSL mesa structure and a corresponding time-integrated PL spectrum measured by $\mu$-TRPL spectroscopy are shown. Several distinct sharp emission lines are clearly resolved in the shaded area of 1,3 , and 5 , which are observed as bright lines in Fig. 1(a). Uniform area outside the masked area gives only broad featureless luminescence and the energy positions of sharp emission lines differ from mesa to mesa, therefore, the observed sharp emission lines are originated from the limited number of localized states randomly distributing in the ZnCdS alloy layers in each mesa structures [8]. Time evolution of PL spectra extracted from the streak image with 10 ps temporal width is summarized in Fig. 2(a). Within 10 ps after excitation, PL lineshape shows rather broad featureless lineshape. In 20 ps after excitation, a sharp line labeled 5 starts to grow and in 40 ps, lower energy peaks of 1 and 3 can be also recognized. This is a direct indication of exciton population in each localized centers, and different built-up time is possibly attributed to the different exciton capture efficiency of each localization sites.

Figure 2(b) shows PL decay curves detected at each shaded energy range denoted in Fig. 1(b). Decay times deduced from the time domain of $t<50 \mathrm{ps}$ after pulse excitation are also given in the figure. It is noted that clear contrast in decay behavior among the energy ranges and a strong correlation between signal intensities in streak image and the observed decay rates can be observed. The bright lines from the localized states show slower decay, while the dark area in between bright lines exhibit faster decay. Therefore, this fast decay behavior observed in these dark regions can be interpreted as the exciton transfer into the localization centers.

The observed fast exciton capture rates into the isolated localized states formed in ZnCdS layers with SPSL structures is very promising features for high speed operation of the SPE, however, it is found that selectively grown ZnCdS mesa-shaped structures and also some of the SPSL samples did not exhibit

any sharp emission lines but only broad luminescence appeared. In order to study the origin of this difference, exciton energy relaxation properties were investigated employing two uniformly grown SPSL structures which represent both types of optical features. In these samples, sharp emission lines are invisible (visible) in sample A (B), which is composed of $2.0 \mathrm{~nm}(0.6 \mathrm{~nm})$ thick ZnCdS and $1.0 \mathrm{~nm}(0.5$ $\mathrm{nm})$ thick MgS layers. Spectrally integrated TRPL measurements revealed that the spectral redshift in 40-60 ps after pulse excitation can be clearly observed in the sample B, while almost identical luminescence was found for all temporal range in the sample A. This spectral shift is possibly attributed to the exciton re-distribution under the effective exciton relaxation processes, which is also supported by the fact that the built-up time of sharp emission lines of $\sim 40$ ps observed in Fig. 1(a) coincides well with the time range of 40-60 ps in which the redshift occurs. Furthermore, these two samples showed clear contrast in the energy dependence of decay profiles as shown in Figs. 3. Noticeably fast decay rate was clearly observed at the higher energy regions in sample B, while the relatively small energy dependence was observed in sample A. Measured decay rates obtained in $t<100 \mathrm{ps}$ time region are plotted as circles (squares) for the sample A (B) in Fig. 4, and these data are analyzed using the theoretical expression $[10,11]$, where the measured lifetime at the photon energy $E$ is described as;

$$
\tau^{-1}(E)=\tau_{r}^{-1}\left\{1+\exp \left(\frac{E-E_{m e}}{E_{0}}\right)\right\}
$$


where $\tau(E)$ is the lifetime measured at the emission energy $E, \tau_{r}$ is the radiative lifetime of the localized excitons, $E_{0}$ is the characteristic energy indicating localization depth and $E_{m e}$ indicates the mobility edge. Fitted curves and the parameters giving the best fit to the experimental data are also shown in the figure.

These obtained results shows that the energy relaxation of excitons into the deeper localization states is substantially induced in the sample B which is composed of shorter period SPSL structures. Lower mobility edge and shallower localization depth obtained in the sample B indicates the highly effective exciton energy relaxation into the deeper localized sites with smaller density of states. This leads to the observation of single-atom-like sharp emission lines from the strongly isolated localized states with stronger zero-dimensionalities.
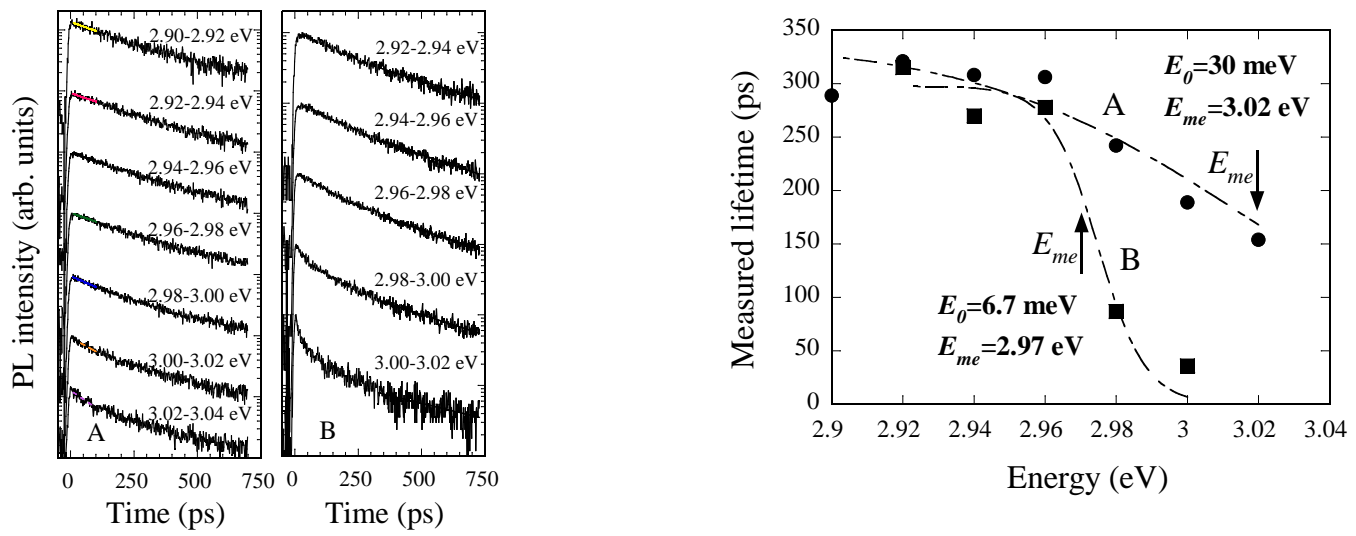

Fig. 3 (left) Energy-dependent decay profiles for two ZnCdS/MgS SPSL samples A and B. In sample B, fast decay components are clearly observed in higher energy regions.

Fig. 4 (right) Photon-energy-dependent measured lifetimes extracted from $t<100$ ps region are plotted as closed circles (squares) for sample A (B). Dot-dashed lines are the theoretical fittings using the Eq. (1). Obtained values of localization depth and the mobility edge for each sample are also shown.

\section{Conclusions}

Dynamical properties of exciton relaxation into the spatially isolated localized centers were investigated. Spatially resolved $\mu$-TRPL measurements revealed that photogenerated excitons relax into spatially isolated localized centers within 40 ps after pulse excitation, which leads to the effective exciton population in the localized states in the presence of short period superlattice structures. It was also revealed that limited number of atom-like emission lines were possible in the samples which have high exciton transfer efficiency to the deeper and therefore strongly 0-D. localization states, which is highly promising for the realization of completely secure single photon sources.

\section{References}

[1] C. Becher, A. Kiraz, P. Michler, A. Imamoglu, W. V. Schoenfeld, P. M. Petroff, Lidong Zhang, and E. Hu, Phys. Rev. Lett. 63, 121312 (2001).

[2] C. Santori, M. Pelton, G. Solomon, Y. Dale, Y. Yamamoto, Phys. Rev. Lett. 86, 1502 (2001).

[3] E. Moreau, I. Robert, J. M. Gerard, I. Abram, L. Manin, and V. Thierry-Mieg, Appl. Phys. Lett. 79, 2865 (2001).

[4] C. H. Bennett, G. Brassard, and A. K. Ekert, Sci. Am. 267, 50 (1992).

[5] G. Brassard, G. Lutkenhaus, T. Mor, and B. C. Sanders, Phys. Rev. Lett. 85, 1330 (2000). 
[6] G. Lutkenhaus, Phys. Rev. A61, 052304 (2000).

[7] J. M. Gerard and B. Gayral, Physica E9, 131 (2001).

[8] H. Kumano, Y. Hitaka, and I. Suemune, Appl. Phys. Lett. 82, 4277 (2003).

[9] A. Ueta, T. Shimozawa, A. Avramescue, I. Suemune, H. Machida, and N. Shimoyama, J. Cryst. Growth 221, 425 (2000).

[10] M. Oueslati, C. Benoit à la Guillaume, and M. Zouaghi, Phys. Rev. B 37, 3037 (1988).

[11] C. Gourdon, and P. Lavallard, phys. stat. sol. (b) 153, 641 (1989). 